\title{
UNRELIABILITY OF LOCAL REACTIONS TO SERUM AS TESTS FOR GENERAL SENSITIVITY
}

\author{
BY \\ L. J. M. LAURENT \\ Honorary Consultant Physician, South East Metropolitan Regional Hospital Board \\ AND \\ H. J. PARISH \\ Clinical Research Director, The Wellcome Research Laboratories
}

The problem of sensitivity tests for serum can be stated to be as follows: Is it possible, by observing the local reaction which follows an intradermal or subcutaneous injection of a small amount of serum, to determine beforehand whether or not a patient will develop anaphylactic shock, and/or severe serum sickness, when given a full therapeutic or prophylactic dose? To be of practical value, the local reaction should show within at least one hour of the test dose, otherwise the doctor would have to postpone the main dose far too long.

We have long maintained that local reactions are unreliable guides of general sensitivity (Laurent and Parish, 1952). A recent paper, however, entitled "An Analysis of Tetanus Prophylaxis in 3,455 Cases" by Binns (1961), though it is a useful collection of data, contains some statements and conclusions in opposition to our views, and they should not be permitted to pass unchallenged. The author claims, on the basis of 9 months' experience at the Leeds Public Dispensary, that the local reaction which follows a subcutaneous injection of $0.1 \mathrm{ml}$. serum is "important and that due cognizance should be taken of it". He has found a "relationship" between the local reaction and the general reaction, and he implies-though he does not say so-that tests for a local reaction could be of value in detecting the presence of general serum-sensitivity.

According to Binns's own figures, 73 patients had a local reaction within an hour, and of these only three developed either anaphylactic shock or serious serum sickness; presumably the other seventy had average or mild serum sickness or had none at all. Local reaction appeared within 4 days in 133 patients, and of these eight developed anaphylactic shock or serious serum sickness; presumably the other 125 had average or mild serum sickness or had none at all. It follows, therefore, that when the tests are read after one hour, a positive result signifies hypersensitivity in only 4 per cent.; when read after 4 days, it signifies hypersensitivity in only 6 per cent. Binns remarks that "the timing of the local reaction seemed to make little difference to the risk of developing a general reaction subsequently". Our comment on this statement is that time is necessarily at the crux of the problem. How can the late appearance of a local reaction (say after 24 hours or more) be helpful as a guide to procedure in administering the main dose of serum?

Between the 5th and the 12th day after the injection of tetanus antitoxin, he has observed a sharp increase in the incidence of local reactions followed immediately by a sharp decrease; there was a similar and almost simultaneous fluctuation in the incidence of general reactions. He remarks on this close correlation and notes the short intervals, in hours, between local and general reactions; it is not clear whether or not he grasps the significance of the observation. These local reactions were obviously part of the serum sickness which normally starts between the 5th and the 12th day. Pain, swelling, redness, and often urticaria, at the site of injection of serum, before, at the same time as, or after the appearance of a serum rash, are of course extremely common, and have been known ever since serotherapy began. These local reactions at the site of injection are often the sole manifestation of the serum disease in mild cases; they are not evidence of a pre-existent sensitivity.,

A lack of correlation between positive intradermal tests and general serum sensitivity has been the experience of one of us (L. J. M. L.) in the course of titration of streptococcal antitoxin on scarlet fever patients. A little over fifty patients, with strongly positive intradermal reactions (mean diameter $20 \mathrm{~mm}$. or more) to a serum dilution of 1 in 10 , had to be given full doses of antitoxin a few hours later; none developed any serious general reaction. 
If it could be shown that anaphylactic shock and severe serum sickness occur only in patients with a positive local reaction to the test, within an hour or two, the test could be of some value. One could deal cautiously with all the positive reactors, for example, by giving them small divided doses of serum, despite the fact that in a large majority of them-96 per cent. in Binns's series-these precautions would be unnecessary. Unfortunately, anaphylactic shock and severe serum sickness have occurred in patients who gave no local reaction to the test. Ratner (1943) described the types of reactions resulting from diagnostic skin tests, and included "Systemic reaction with negative local reaction. Here no local reaction occurs, but within a few to 15 or 20 minutes the individual develops severe dyspnoea, without other symptoms. Such a case is particularly hazardous and may terminate fatally". Rolleston and Ronaldson (1940) stated that "it must be recognized that (sensitivity) tests do not invariably furnish the required information; quite severe immediate reactions have been met in patients who gave no response to the tests". Park (1924) also warned that "persons who do not possess skin hypersensitiveness may occasionally develop serious symptoms after an intravenous and even after an intra-muscular injection of horse serum".

It follows, therefore, that both "positive" and "negative" results are unreliable guides before serum is administered. The "trial dose" or "test dose for general sensitivity", which we advocate, consists in giving a small amount of serum or serum-dilution subcutaneously, thus ensuring a slow absorption into the general circulation, and in observing the patient for the occurrence of a general reaction. Should anaphylaxis occur under the circumstances, it is likely to be mild and respond quickly to treatment. The local reaction is ignored. Ideally, the period of observation should be 2 hours; we know from experience that anaphylactic shock which starts after 2 hours is very rare. The half-hour period is a compromise; the first half-hour is by far the most dangerous period. Binns had two cases of severe anaphylaxis, one 30 minutes after the test dose, and the other 50 minutes after the test dose and 20 minutes after the full dose. These cases confirm our views, and demonstrate the value of the method and also its limitations.
Binns reports 27 general reactions out of 2,726 injections-approximately 1 per cent. This incidence is slightly less than one would expect from prophylactic doses of tetanus antitoxin; the antihistaminic drugs which were administered probably contributed to the low incidence. His results show the risks from prophylaxis by tetanus antitoxin and also confirm that these risks are not unduly alarming. His statement that we [Laurent and Parish (1952)] suggested that "anaphylactic reactions occurred in 1 in 50,000 to 200,000 cases" is inaccurate. We were quoting the observations of previous workers, and the figures referred to deaths from anaphylaxis.

With regard to the incidence of serum reaction in patients suffering from asthma or infantile eczema, Binns mentions nine patients who had no reaction at all. This is not surprising in view of the short period of observation, namely, 9 months. One of us (L. J. M. L.), over a period of 37 years of experience of sero-therapy, can remember about twenty patients with either asthma or eczema who suffered no illeffects after serum. On the other hand, he also remembers two fatal cases of anaphylaxis:

(1) A child with asthma was given a large dose of diphtheria antitoxin without a preliminary triab dose. The doctor was under the impression that the laboured breathing was due to "croup".

(2) A child with infantile eczema and scarlet fever was given a dose of streptococcal antitoxin without a preliminary trial dose. The doctor was not aware of the special risks of injecting serum into an allergic child.

Neither patient had been given serum before.

\section{CONCLUSION}

The local reaction to serum, whether "positive" or "negative", is of no practical significance as an indication of general sensitivity. The value of the "trial dose" subcutaneously as a test for general sensitivity is reaffirmed and its limitations defined.

\section{REFERENCES}

Binns, P. M. (1961). Brit. J. prev. soc. Med., 15, 180.

Laurent, L. J. M., and Parish, H. J. (1952). Brit. med. J., $1,1294$.

Park, W. H. (1924). J. Immunol., 9, 17.

Ratner, B. (1943). "Allergy, Anaphylaxis and Immunotherapy". Williams and Wilkins, Baltimore.

Rolleston, J. D., and Ronaldson, G. W. (1940). "Acute Infectious Diseases", 3rd ed. Heinemann, London. 